\title{
Adaptive and robust techniques (ART) for thermoacoustic and photoacoustic tomography
}

Yao Xie, Bin Guo, Jian Li, Geng Ku, Lihong V. Wang

Yao Xie, Bin Guo, Jian Li, Geng Ku, Lihong V. Wang, "Adaptive and robust techniques (ART) for thermoacoustic and photoacoustic tomography," Proc. SPIE 6437, Photons Plus Ultrasound: Imaging and Sensing 2007: The Eighth Conference on Biomedical Thermoacoustics, Optoacoustics, and Acoustooptics, 643715 (13 February 2007); doi: 10.1117/12.698391 


\title{
Adaptive and Robust Techniques (ART) for Thermoacoustic and Photoacoustic Tomography*
}

\author{
Yao Xie ${ }^{a}$, Bin $\mathrm{Guo}^{a}$, Jian $\mathrm{Li}^{a}$, Geng $\mathrm{Ku}^{b}$ and Lihong V. Wang ${ }^{b}$ \\ ${ }^{a}$ Electrical and Computer Engineering Department, University of Florida, \\ Gainesville, Florida, 32611, U.S.A.; \\ ${ }^{b}$ Department of Biomedical Engineering, Texas A\&M University, \\ 234C Zachary Engineering Center, College Station, U. S. A.
}

\begin{abstract}
In this paper, we present new Adaptive and Robust Techniques (ART) for microwave-based thermoacoustic tomography (TAT) and laser-based photo-acoustic tomography (PAT), and study their performances for breast cancer detection. TAT and PAT are emerging medical imaging techniques that combine the merits of high contrast due to electromagnetic or laser stimulation and high resolution offered by thermal acoustic imaging. The current image reconstruction methods used for TAT and PAT, such as the widely used Delay-and-Sum (DAS) approach, are data-independent and suffer from low resolution, high sidelobe levels, and poor interference rejection capabilities. The data-adaptive ART can have much better resolution and much better interference rejection capabilities than their data-independent counterparts. By allowing certain uncertainties, ART can be used to mitigate the amplitude and phase distortion problems encountered in TAT and PAT. Specifically, in the first step of ART, RCB is used for waveform estimation by treating the amplitude distortion with an uncertainty parameter. In the second step of ART, a simple yet effective peak searching method is used for phase distortion correction. Compared with other energy or amplitude based response intensity estimation methods, peak searching can be used to improve image quality with little additional computational costs. Moreover, since the acoustic pulse is usually bipolar: a positive peak, corresponding to the compression pulse, and a negative peak, corresponding to the rarefaction pulse, we can further enhance the image contrast in TAT or PAT by using the peak-to-peak difference as the response intensity for a focal point. The excellent performance of ART is demonstrated using both simulated and experimentally measured data.
\end{abstract}

Keywords: Thermoacoustic Tomography, Adaptive and Robust Techniques

\section{INTRODUCTION}

Thermoacoustic tomography (TAT) and photoacoustic tomography (PAT) are emerging technologies that are promising in a wide span of biomedical applications. ${ }^{1-5}$ The physical basis of TAT lies in the contrast of the radiation absorption rate among different biological tissues, and PAT is based on the contrast of the optical property among different tissues. Due to the thermoacoustic effect, when a short electromagnetic pulse (microwave in TAT or laser in PAT) is absorbed by the tissue, the heating results in expansion which generates acoustic signals. In TAT or PAT, an image of the tissue absorption or optical properties is reconstructed from the recorded thermoacoustic signals. Such an image reveals the physiological and pathological status of the tissue, which has been used in many applications including breast cancer and tumor-related vasculature detections.

An attractive feature of TAT and PAT is that they combines the fine imaging resolution and good spatial contrast. The thermoacoustic and photoacoustic images are reconstructed from the ultrasonic signals, which

This research was supported in part by the National Institutes of Health (NIH) Grant No. 1R41CA107903-1.

Further author information: (Send correspondence to Jian Li)

Jian Li: E-mail: li@dsp.ufl.edu, Telephone: (352) 392-2642

Yao Xie: E-mail: yaoxie@stanford.edu, Telephone: (352) 871-0826

Bin Guo: E-mail: guobin@dsp.ufl.edu, Telephone: (352) 392-5241

Geng Ku: E-mail: gku@oilab.tamu.edu

Lihong V. Wang: E-mail: lhwang@biomed.wustl.edu, Telephone: (314) 935-6152

Photons Plus Ultrasound: Imaging and Sensing 2007: The Eighth Conference on Biomedical Thermoacoustics, Optoacoustics, and Acousto-optics, edited by Alexander A. Oraevsky, Lihong V. Wang,

Proc. of SPIE Vol. 6437, 643715, (2007) · 1605-7422/07/\$18 - doi: 10.1117/12.698391

Proc. of SPIE Vol. 6437 643715-1 


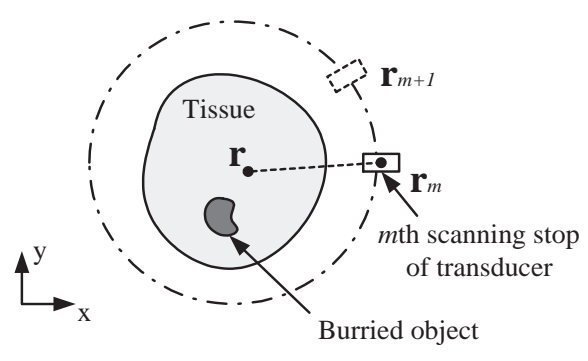

(a)

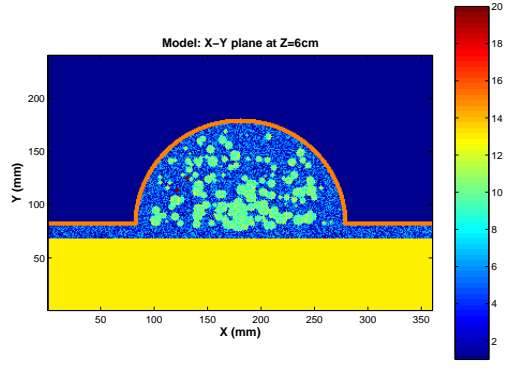

(b)

Figure 1. (a): A schematic of a 2-D synthetic aperture based TAT scanning system; (b): the dielectric property distribution of the simulated 2-D breast model.

means that they possess good spatial resolution as pure ultrasound imaging. Microwave-induced TAT takes advantage the high contrast of biological tissue in electromagnetic frequency band. Lase-induced PAT makes use of the fact that at some wavelengths of light, the absorption coefficient of blood can be ten times higher than that of its surrounding tissues. TAT has deeper penetration into the biological tissues than PAT. However, PAT can reach deeper structures of tissues by using NIR excitation sources and proper contract agents. ${ }^{5}$

One of the key challenges in TAT and PAT is to develope accurate and robust image reconstruction methods. Time-domain approximate reconstruction algorithms such as the $\mathrm{DAS}^{6}$ (weighted or unweighted) type of dataindependent approaches are widely used. They need little prior information on the tissue for image reconstruction and can be fast and simple to implement to process the wideband acoustic signals. Although not based on the exact solution, they provide similar image qualities to those of the exact reconstruction algorithms. However, these data-independent methods suffer from poor resolution and high sidelobe level problems. Data-adaptive approaches, such as the recently introduced Robust Capon Beamforming (RCB) method, ${ }^{7}$ can have much better resolution and much better interference rejection capability than their data-independent counterparts.

A common assumption of the existing methods is that the surrounding tissue is acoustically homogeneous. This approximation is inadequate in many medical imaging applications. For example, the sound speed in human female breast varies widely from $1430 \mathrm{~m} / \mathrm{s}$ to $1570 \mathrm{~m} / \mathrm{s}$ around the commonly assumed speed of 1510 $\mathrm{m} / \mathrm{s}^{8}$ The heterogeneous acoustic properties of biological tissues cause amplitude and phase distortions in the recorded acoustic signals, which can result in significant degradations in image quality. ${ }^{9}$ Although amplitude and phase distortion of biological tissue has been studied extensively in Ultra-sound Tomography (UT), however, the problems in TAT and TAT are somewhat different from that in UT. For example, in TAT, for the breast tissue with a relatively weak heterogeneity, phase distortion dominates amplitude distortion. ${ }^{9}$

We propose Adaptive and Robust Techniques (ART) based on RCB for TAT and PAT. ART can be used to mitigate the amplitude and phase distortion problems in TAT and PAT by allowing certain uncertainties. We will demonstrate the excellent performance of ART by using both data simulated on a 2-D breast model and data experimentally measured from mastectomy specimen and blood object sample.

\section{PROBLEM FORMULATION}

Consider a TAT or PAT imaging system as shown in Figure 1(a). A stimulating microwave or laser pulse is absorbed by the biological tissue under testing, which causes a sudden heat change. Due to the thermoacoustic effect, an acoustic pulse is generated which can be recorded by an ultrasonic transducer array (a real aperture array or a synthetic aperture array). The number of transducers in the array (or the number of transducer data acquisition locations in the synthetic aperture array) is $M$. Each transducer is assumed to be omnidirectional.

The data model for the sampled and digitized acoustic signal recorded by the $m$ th transducer is given by:

$$
x_{m}(n)=s_{m}(n)+\tilde{e}_{m}(n), \quad m=1, \cdots, M .
$$


where $n$ is the discrete time index, starting from $t_{0}$ after excitation pulse. The scalar $s_{m}(n)$ denotes the signal component, which corresponds to the acoustic pulse generated at a focal point, and $\tilde{e}_{m}(n)$ is the residual term, which includes unmodelled noise and interference.

The goal of ART is to reconstruct an image of thermoacoustic response intensity $I(\mathbf{r})$, which is directly related to the absorption or optical properties of the tissue, from the recorded data set $\left\{x_{m}(n)\right\}$. Herein the vector $\mathbf{r}$ denotes the focal point location coordinate. To form an image, we scan the focal point at location $\mathbf{r}$ to cover the entire cross-section of the tissue.

The discrete arrival time of the pulse (for the $m$ th transducer) can be determined approximately as:

$$
t_{m}(\mathbf{r})=\left\lfloor-\frac{t_{0}}{\Delta t}+\frac{\left\|\mathbf{r}-\mathbf{r}_{m}\right\|}{\Delta t v_{0}}\right\rfloor .
$$

We will omit the dependence of the arrival time $t_{m}(\mathbf{r})$ on $\mathbf{r}$ hereafter for notational simplicity. Here $\Delta t$ is the sampling interval, and the 3-D vector $\mathbf{r}_{m}$ denotes the location of the $m$ th transducer. The sound speed $v_{0}$ is chosen to be the average sound speed of the biological tissue under interrogation. The notation $\|\mathbf{x}\|$ denotes the Euclidean norm of $\mathbf{x}$, and $\lfloor y\rfloor$ stands for rounding to the greatest integer less than $y$.

The signal components $\left\{s_{m}(n)\right\}_{m=1}^{M}$ are approximately scaled and shifted versions of a nominal waveform $s(t)$ at the source:

$$
s_{m}(n) \approx \frac{\exp \left(-\alpha\left\|\mathbf{r}-\mathbf{r}_{m}\right\|\right)}{\left\|\mathbf{r}-\mathbf{r}_{m}\right\|} \cdot s\left(n-t_{m}\right),
$$

where $\alpha$ is the attenuation coefficient in Nepers $/ \mathrm{m}$. We preprocess the data to time delay all the signals from the focal point $\mathbf{r}$ and compensate for the loss in amplitude due to propagation decay. Let $y_{m}(n)$ denote the signal after preprocessing to backpropagate the detected signal to the source:

$$
y_{m}(n)=\exp \left(\alpha\left\|\mathbf{r}-\mathbf{r}_{m}\right\|\right) \cdot\left\|\mathbf{r}-\mathbf{r}_{m}\right\| x_{m}\left(n+t_{m}\right) ;
$$

then the received vector data model can be written as:

$$
\mathbf{y}(n)=\mathbf{a}_{0} s(n)+\mathbf{e}(n), \quad n=-N, \cdots, N,
$$

where $\mathbf{a}_{0}$ is the corresponding steering vector, which is approximately equal to $\overline{\mathbf{a}}=[1, \cdots, 1]^{T}$,

$\mathbf{y}(n)=\left[y_{1}(n), \cdots, y_{M}(n)\right]^{T}, \mathbf{e}(n)$ represents the noise and interference term after preprocessing, and $(\cdot)^{T}$ denotes the transpose. The time interval of interests for the signal $\mathbf{y}(t)$ is from $-N$ to $N$, which is large enough to covers the expected signal duration in the region of interest.

In reality, both the amplitude and the phase (or pulse arrival time) of the acoustic pulse will be distorted. Amplitude distortion is mainly caused by multi-path in the heterogeneous medium. Phase distortion is due to the nonuniform sound speed, an inaccurate estimate of $t_{0}$, and the transducer calibration error. These distortions will blur the image, raise the image background noise level, and lower the values of the object of interest. ${ }^{9}$

In ART, we mitigate the effects of these distortions by allowing $\mathbf{a}_{0}$ to belong to an uncertainty set centered at $\overline{\mathbf{a}}$ and by considering the signal arriving within the interval from $-N$ to $N$. Our ART algorithm consists of three steps: Step I, Robust Capon Beamforming $(\mathrm{RCB})^{7}$ for robust waveform estimation; Step II, peak-searching for phase aberration mitigation; Step III, intensity calculation for forming the final images.

\section{STEP I OF ART: WAVEFORM ESTIMATION}

The first step of ART is to estimate the waveform of the acoustic pulse generated by the focal point at location $\mathbf{r}$, based on the data model in (5). It will appear that we have neglected the presence of phase distortion by using this data model in the first step. However, by allowing $\mathbf{a}_{0}$ to be uncertain, we can tolerate some phase distortions as well. This approximation causes little performance degradation to our robust algorithm. 
Covariance fitting based $\mathrm{RCB}^{7}$ is used to first estimate the steering vector $\mathbf{a}_{0}$, and use the estimated $\mathbf{a}_{0}$ to obtain an optimal beamformer weight vector for pulse waveform estimation. By assuming that the true steering vector lies in the vicinity of the nominal steering vector $\overline{\mathbf{a}}$, we consider the following optimization problem: ${ }^{7}$

$$
\begin{array}{lll}
\max _{\sigma^{2}, \mathbf{a}_{0}} \sigma^{2} \quad \text { subject to } & \hat{\mathbf{R}}-\sigma^{2} \mathbf{a}_{0} \mathbf{a}_{0}^{T} \geq 0 \\
& \left\|\mathbf{a}_{0}-\overline{\mathbf{a}}\right\|^{2} \leq \varepsilon
\end{array}
$$

where $\sigma^{2}$ is the power of the signal of interest, and

$$
\hat{\mathbf{R}}=\frac{1}{2 N+1} \sum_{n=-N}^{N} \mathbf{y}(n) \mathbf{y}^{T}(n)
$$

is the sample covariance matrix. The second constraint in (6) is a spherical uncertainty set; an elliptical uncertainty set can be used instead if a tighter constraint is desirable. ${ }^{7}$

The parameter $\varepsilon$ in (6) determines the size of the uncertainty set and is a user parameter. To avoid the trivial solution of $\mathbf{a}_{0}=0$, we require that

$$
\varepsilon<\|\overline{\mathbf{a}}\|^{2} .
$$

To attain high resolution and to effectively suppress interference, $\varepsilon$ should be made as small as possible. On the other hand, the smaller the sample size $N$ or the larger the distortions, the larger should $\varepsilon$ be chosen. ${ }^{7}$ Since the performance of RCB does not depend very critically on the choice of $\varepsilon$, in our examples we choose certain reasonable initial values for $\varepsilon$, and then make some adjustments empirically based on image quality.

By using the Lagrange multiplier method, the solution to (6) is given by: ${ }^{7}$

$$
\hat{\mathbf{a}}_{0}=\overline{\mathbf{a}}-[\mathbf{I}+\mu \hat{\mathbf{R}}]^{-1} \overline{\mathbf{a}}
$$

where $\mathbf{I}$ is the identity matrix, $\mu \geq 0$ is the corresponding Lagrange multiplier that can be solved from the following equation:

$$
\left\|(\mathbf{I}+\mu \hat{\mathbf{R}})^{-1} \overline{\mathbf{a}}\right\|^{2}=\varepsilon .
$$

Consider the eigendecomposition on the sample covariance matrix $\hat{\mathbf{R}}$ :

$$
\hat{\mathbf{R}}=\mathbf{U} \boldsymbol{\Gamma} \mathbf{U}^{T},
$$

where the columns of $\mathbf{U}$ are the eigenvectors of $\hat{\mathbf{R}}$ and the diagonal matrix $\boldsymbol{\Gamma}$ consists of the corresponding eigenvalues $\gamma_{1} \geq \gamma_{2} \geq \cdots \geq \gamma_{M}$. Let $\mathbf{b}=\mathbf{U}^{T} \overline{\mathbf{a}}$, where $b_{m}$ denotes its $m$ th element. Then (10) can be rewritten as

$$
\mathcal{L}(\mu)=\sum_{m=1}^{M} \frac{\left|b_{m}\right|^{2}}{\left(1+\mu \gamma_{m}\right)^{2}}=\varepsilon .
$$

Note that $\mathcal{L}(\mu)$ is a monotonically decreasing function of $\mu$, with $\mathcal{L}(0)>\varepsilon$ by $(8)$ and $\lim _{\mu \rightarrow \infty} \mathcal{L}(\mu)=0<\varepsilon$, which means that $\mu$ can be solved efficiently, say, by using the Newton's method (see ${ }^{7}$ for more details). After obtaining the value of $\mu$, the estimate $\hat{\mathbf{a}}_{0}$ of the actual steering vector $\mathbf{a}_{0}$ is determined by (9).

Observe that there is a "scaling ambiguity" in (6) by treating both the signal power $\sigma^{2}$ and the steering vector $\mathbf{a}_{0}$ as unknowns $\left(\mathrm{see}^{7}\right)$. To eliminate this ambiguity, we scale the solution $\hat{\mathbf{a}}_{0}$ to make its norm satisfy the following condition:

$$
\left\|\hat{\mathbf{a}}_{0}\right\|^{2}=M
$$


To obtain an estimate for the signal waveform $s(n)$, we apply a weight vector to the preprocessed signals $\{\mathbf{y}(n)\}_{n=-N}^{N}$. The weight vector is determined by using the estimated steering vector $\hat{\mathbf{a}}_{0}$ in the weight vector expression of the standard Capon beamformer (see, e.g., ${ }^{7}$ ):

$$
\hat{\mathbf{w}}_{\mathrm{RCB}}=\frac{\left\|\hat{\mathbf{a}}_{0}\right\|}{M^{1 / 2}} \cdot \frac{\left[\hat{\mathbf{R}}+\frac{1}{\mu} \mathbf{I}\right]^{-1} \overline{\mathbf{a}}_{0}}{\overline{\mathbf{a}}_{0}^{T}\left[\hat{\mathbf{R}}+\frac{1}{\mu} \mathbf{I}\right]^{-1} \hat{\mathbf{R}}\left[\hat{\mathbf{R}}+\frac{1}{\mu} \mathbf{I}\right]^{-1} \overline{\mathbf{a}}_{0}} .
$$

Note that (14) has a diagonal loading form, which allows the sample covariance matrix to be rank-deficient. The beamformer output can be written as:

$$
\hat{s}_{\mathrm{RCB}}(n)=\hat{\mathbf{w}}_{\mathrm{RCB}}^{T} \mathbf{y}(n), \quad n=-N, \cdots, N
$$

which is the waveform estimate for the acoustic pulse generated at the focal point at location $\mathbf{r}$.

RCB can provide a much better waveform estimate than the conventional DAS but at a higher computational cost. For a single focal point, RCB requires $O\left(M^{3}\right)$ flops, which mainly comes from the eigen-decomposition of the sample covariance matrix $\hat{\mathbf{R}} ;{ }^{7}$ DAS needs only $O(M)$ flops. DAS can be used as a fast image reconstruction method to provide initial imaging results.

The weight vector used by DAS for waveform estimation is

$$
\hat{\mathbf{w}}_{\mathrm{DAS}}=\overline{\mathbf{a}}
$$

and the estimated waveform is given by

$$
\hat{s}_{\mathrm{DAS}}(n)=\hat{\mathbf{w}}_{\mathrm{DAS}}^{T} \mathbf{y}(n)=\sum_{m=1}^{M} y_{m}(n), \quad n=-N, \cdots, N .
$$

\section{STEP II OF ART: PEAK SEARCHING}

Based on the estimated waveform obtained in Step I for the focal point at location $\mathbf{r}$, in Step II of ART, we will search for the two peaks of the bipolar acoustic pulse generated by the focal point. In a homogeneous background we can accurately calculate the arrival time of the acoustic pulse generated by the focal point at location $\mathbf{r}$ by using (2); this is not true in heterogeneous biological tissues due to the nonuniform sound speed. We can assume that the original peak remains a peak in the waveform estimated from Step I of ART, since the amplitude distortion is not severe in $\mathrm{TAT}^{9}$ (similar for PAT).

The bipolar acoustic pulse has one peak positive and another negative. We determine the positive and negative peak values as follows:

$$
\begin{aligned}
& P^{+}=\max \left\{\max _{n \in[-\Delta, \Delta]} \hat{s}(n), 0\right\}, \\
& P^{-}=\min \left\{\min _{n \in[-\Delta, \Delta]} \hat{s}(n), 0\right\},
\end{aligned}
$$

where the searching range $[-\Delta, \Delta] \in[-N, N]$ is around the calculated arrival time given by $(2)$. Here $\Delta$ is a user parameter. Since the peak searching is independent of the particular waveform estimation methods, we use $\hat{s}(n)$ to denote the waveform estimated by either DAS or ART.

The search range is determined by the difference between the true arrival time $\bar{t}_{m}$ and the calculated arrival time $t_{m}$ based on (2). An expression the arrival time difference has been given in ${ }^{9}$ in the following form:

$$
\delta_{m}\left(\mathbf{r}^{\prime}\right)=\bar{t}_{m}-t_{m} \propto \frac{\left[v\left(\mathbf{r}^{\prime}\right)-v_{0}\right]}{v_{0}}
$$


where $\mathbf{r}^{\prime}$ is a point within the line connecting the focal point at location $\mathbf{r}$ and the $m$ th transducer at location $\mathbf{r}_{m}$, and $v\left(\mathbf{r}^{\prime}\right)$ is the local sound speed. In (20), the higher order terms of $\left[v\left(\mathbf{r}^{\prime}\right)-v_{0}\right] / v_{0}$ have been ignored. It is reasonable to assume that $v\left(\mathbf{r}^{\prime}\right)$ is Gaussian distributed with mean $v_{0}$ and variance $\sigma_{v}^{2}$. Consequently the arrival time difference is also Gaussian distributed with zero-mean and variance $\sigma_{\delta}^{2} \propto \sigma_{v}^{2} / v_{0}^{2}$. If we choose $\Delta=\sigma_{\delta}$, and the duration of the acoustic pulse is $\tau$, we can find the two peaks of the pulse within the interval $\left(-\sigma_{\delta}, \sigma_{\delta}+\tau\right)$ on the recorded signals with a high probability of 0.6826 . This analysis is consistent with the experimental measurements in. ${ }^{10}$ From our examples, we found that a symmetric range $[-\Delta, \Delta]$ around the estimated arrival time performs similarly to the asymmetric range $[-\Delta, \Delta+\tau]$, and we use the former since it is easy to handle in practice. Also, we can use similar techniques as those in ${ }^{10}$ to estimate $\sigma_{\delta}$ to find a good searching range for Step II of ART, and to estimate $\tau$ for the energy type methods in our examples.

There is a tradeoff in choosing the searching range. The larger the searching range, the higher the probability we can find the peaks of the acoustic pulse within the range. However, if the range is chosen too large, the interferences may cause false peaks, and as a consequence, we are more likely to find a false peak. In our examples we choose the best searching range empirically based on the estimates of $\hat{\sigma}_{\delta}$.

\section{STEP III OF ART: INTENSITY CALCULATION}

Based on the estimation of the waveform generated by the focal point at location $\mathbf{r}$, the response intensity for need to be calculated. There are two major types of response intensity measurement approaches: amplitude based and energy based. They extract different information from the estimated waveform, which may be useful to physicians in different ways.

Conventional DAS uses the amplitude based measure for TAT imaging, ${ }^{6}$ with the corresponding response intensity given by $\hat{s}(0)$, or equivalently:

$$
I_{\mathrm{C}}=\hat{s}(0)=\sum_{m=1}^{M} y_{m}(0) .
$$

The energy based measure, such as the one used in the mono-static and multi-static microwave imaging for breast cancer detection, ${ }^{11}$ calculate the intensity by:

$$
I_{\mathrm{E}}=\sum_{n=0}^{\tau} \hat{s}^{2}(n)=\sum_{n=0}^{\tau}\left[\sum_{m=1}^{M} y_{m}(n)\right]^{2},
$$

We can consider using the peak value as the response intensity measure due to the bipolar nature of the response at the focal point:

$$
I_{\mathrm{P}}= \begin{cases}P^{+} & \text {if }\left|P^{+}\right| \geq\left|P^{-}\right| \\ P^{-} & \text {otherwise }\end{cases}
$$

Herein we keep the sign of the maximum amplitude since the sign of the peak may also contain some information about the focal point.

Peak-searching maximizes the output signal-to-noise ratio. An intuitive explanation is that, given the fact that the acoustic pulse is bipolar, ${ }^{6}$ if we assume that the residual term $\mathbf{e}(t)$ is stationary, or its power is uniform over time, then the signal-to-noise ratio is maximized at the (positive or negative) peak of the acoustic pulse. As a comparison, the conventional DAS (21) fixes the samples to be summed up at the calculated arrival time. Due to phase distortions, the waveform at the calculated time may be far from the peak value.

We can also employ peak-to-peak difference as the response intensity for the focal point at location $\mathbf{r}$ :

$$
I_{\mathrm{PP}}=P^{+}-P^{-} \geq 0 .
$$

Peak-to-peak difference has higher imaging contrast than peak value measure: the peak-to-peak difference of the bipolar pulse is approximately twice the absolute peak value, which means that the output signal power of the former is four times of the latter; yet the noise power of the former is only twice of the latter. Therefore the output Signal-to-Noise Ratio (SNR) is doubled by using the peak-to-peak difference rather than the peak value. Both peak-value and peak-to-peak difference measures belong to the amplitude based measures. 


\section{NUMERICAL AND EXPERIMENTAL EXAMPLES}

We demonstrate the performance of ART using both numerically simulated and experimentally measured TAT and PAT data. The ART images are compared with the DAS images.

\subsection{Numerical Examples}

To test TAT imaging for breast cancer detection, we simulate a 2-D cylindrical breast model using the FiniteDifference Time-Domain (FDTD) method. The 2-D breast model includes $2 \mathrm{~mm}$ thick skin, chest wall, as well as randomly distributed fatty breast tissues and glandular tissues. The cross-section of the breast model is a half circle with a $10 \mathrm{~cm}$ diameter. We place two closely located $2 \mathrm{~mm}$-diameter tumors at $x=13.0 \mathrm{~cm}, y=12.1$ $\mathrm{cm}$, and at $x=12.4 \mathrm{~cm}, y=11.5 \mathrm{~cm}$, with a 5 - $\mathrm{mm}$ spacing between them.

The dielectric properties of the breast tissues are assumed to be Gaussian random variables with variations of $\pm 10 \%$ around their nominal values. The nominal values are chosen to be typical of those reported in the literature, which is given in Table 1 of. ${ }^{11}$ The dielectric property distribution of the 2-D simulated breast model in shown in Figure 1(b). The dispersive properties of the tissues are also considered in the model. Fat breast tissues and glandular tissues are randomly distributed in the breast model. The differential equations in ${ }^{12}$ are used to characterize the thermoacoustic effect in our FDTD simulations.

We model the sound speed within the breast as a Gaussian random variable with variation $\pm 5 \%$ around the assumed average sound speed of $1500 \mathrm{~m} / \mathrm{s}$. The probing microwave pulse used here is a modulated rectangular pulse with a modulating frequency of $800 \mathrm{MHz}$. The duration of the pulse is $1 \mu \mathrm{s}$. Before applying the aforementioned preprocessing steps and ART, we remove the strong skin response using techniques similar to those in. ${ }^{11}$ In the following all the images are displayed on a linear scale, and we will name the imaging methods by their waveform estimation method followed by the intensity calculation approach, such as "DAS-C."

Figure 2 shows the images for the simulated breast model with two $2 \mathrm{~mm}$ - diameter tumors, spaced $5 \mathrm{~mm}$ away from each other. For this example we use $\varepsilon=0.1 M$ and searching range $[-3,3]$. For this case, none of the DAS methods can resolve these two closely located tumors. Here we just show one DAS image, the DAS-PP image, in Figure 2(a). RCB-E2, in Figure 2(b), cannot resolve the tumors either. Both APT-P and APT-PP can resolve the two tumors, as shown in Figures 2(c) and 2(d), respectively. Close examinations of the images for RCB-E2 and ART-PP, shown in Figures 2(e) and 2(f), demonstrate the high resolution of ART.

\subsection{Experimental Results}

We have also tested ART and DAS on TAT experimental data obtained from a mastectomy specimen, ${ }^{5}$ and on PAT experimental data obtained from a blood object sample ${ }^{5}$ by the Optical Imaging Laboratory at the Texas A\&M University.

In the TAT experiment, the breast specimen was formed to a cylindrical shape inside a plastic bowl. The bowl was immersed in ultrasound coupling medium in a container. The acoustic signals were recorded at $M=160$ equally spaced scanning stops on a circular track of radius $9.7 \mathrm{~cm}$. The thickness of this specimen was about 9 $\mathrm{cm}$ in a round plastic bowl of $11 \mathrm{~cm}$ in diameter. The lesion in the specimen was diagnosed as infiltrating lobular carcinoma; the size of the tumor was about $20 \mathrm{~mm} \times 12 \mathrm{~mm}$ on TAT image, and about $26 \mathrm{~mm} \times 15 \mathrm{~mm}$ on the radiography ( $\mathrm{see}^{5}$ for more details). Figure 3 shows the reconstructed images for the breast specimen. In the following images, the searching range was set to [-120, 120], and $\varepsilon=0.5 M$ for all the RCB used herein. In Figure $3(\mathrm{a})$, for DAS-C, the true tumor is barely identifiable from the surrounding clutters. The DAS-E1 shown in Figure 3(b) and the DAS-E2 shown in Figure 3(c) provide higher imaging contrast than DAS-C but show strong clutter. In Figure 3(d), for RCB-E2, a false tumor shows up, which demonstrates the need for robustness in the presence of relatively strong phase distortion. DAS-P is shown in Figure 3(e) and ART-P is shown in Figure 3(f). DAS-PP and ART-PP produce the best images in Figures 3(g) and 3(h), respectively, with the location and shape of the tumor consistent with the radiograph in Figure 3(i). ${ }^{5}$ If we define the Signal-to-Background Ratio (SBR) (i.e., squaring the pixel values of the image, the ratio of the maximum to the total sum of the squared values) as a image quality measurement metric, ART-PP has an SBR twice that of DAS-PP, which means a 3 -dB gain for ART-PP. For comparison, the image formed by the exact inverse solution of TAT (see ${ }^{5}$ for more details) is shown in Figure 3(j). 
In the PAT experiment, a raw blood object mimicking blood vessel was embedded in a $1.5 \mathrm{~mm}$ diameter chicken breast muscle tube at a depth of $4 \mathrm{~cm}$ away from the laser illuminated tissue surface ( $\mathrm{see}^{5}$ for details). The number of scanning top is $M=120$. In the DAS imaging results by $\mathrm{Ku}$ et al.,$^{5} 4 \mathrm{~cm}$ is the maximum depth at which the blood could still be clearly visible. The DAS image is shown in 4(a), which was spoiled by some noticeable interferences. In the following images, the searching range was set to $[-5,5]$, and $\varepsilon=0.8 M$ for all the RCB used herein. To show the detailed structure, Figure 4 is normalized and displayed in logarithmic scale with dynamic range $15 \mathrm{~dB}$. RCB-E2, as shown in Fig. 4(b), can remove the interferences, but the image of blood object is also blurred due to the effects of phase distortions. DAS-PP, shown in Fig. 4(e), enhances the image contrast but cannot remove the strong interferences. APT-PP can effectively suppress the interferences and preserve the image of blood object, although the blood object image becomes somewhat faint since ART-PP puts much effort on suppressing the strong interferences.

\section{CONCLUSIONS}

Adaptive and Robust Techniques (ART) have been proposed for thermoacoustic tomography and photoacoutic tomography. ART is robust to the amplitude and phase distortions in the recorded signals caused by the acoustic heterogeneity of biological tissues. ART consists of three steps: in the first step, ART uses the data-adaptive Robust Capon Beamforming (RCB) for waveform estimation; in the second step of ART, a simple yet effective peak searching method is used to mitigate the phase distortion in the estimated waveform; in the third step, the response intensity is calculated for the focal point using various approaches, among which the peak-topeak difference measure further enhances the image contrast. Examples based on a numerically simulated 2-D breast model and two sets of experimentally measured data demonstrate the excellent performance of ART: high resolution, low-side lobe level, and much improved interference suppression capability.

\section{REFERENCES}

1. R. A. Kruger, P. Liu, Y. R. Fang, and C. R. Appledorn, "Photoacoustic ultrasound (PAUS) - reconstruction tomography," Med. Phys., vol. 22, pp. 1605-1609, Oct. 1995.

2. R. A. Kruger, K. K. Kopecky, A. M. Aisen, D. R. Reinecke, G. A. Kruger, and W. L. Kiser, "Thermoacoustic ct with radio waves: a medical imaging paradigm," Radiology, vol. 211, pp. 275-278, Apr. 1999.

3. L. V. Wang, X. Zhao, H. Sun, and G. Ku, "Microwave-induced acoustic imaging of biological tissues," Med. Phys., vol. 70, pp. 3744-3748, Sept. 1999.

4. R. A. Kruger, K. D. Miller, H. E. Reynolds, W. L. Kiser, D. R. Reinecke, and G. A. Kruger, "Breast cancer in vivo: Contrast enhancement with thermoacoustic CT at $434 \mathrm{MHz}$ - feasibility study," Radiology, vol. 216, pp. 279-283, 2000.

5. G. Ku, B. D. Fornage, X. Jin, M. Xu, K. K. Hunt, and L. V. Wang, "Thermoacoustic and photoacoustic tomography of thick biological tissues toward breast imaging," Technology in Cancer Research \& Treatment, vol. 4, pp. 1-7, Oct. 2005.

6. C. G. A. Hoelen and F. F. M. de Mul, "Image reconstruction for photoacoustic scanning of tissue structures," Applied Optics, vol. 39, no. 31, pp. 5872-5883, 2000.

7. J. Li, P. Stoica, and Z. Wang, "On robust Capon beamforming and diagonal loading," IEEE Transactions on Signal Processing, vol. 51, pp. 1702-1715, July 2003.

8. T. Szabo, Diagnostic Ultrasound Imaging: Inside Out. Academic Press, Sept. 2004.

9. Y. Xu and L. V. Wang, "Effects of acoustic heterogeneity in breast thermoacoustic tomography," IEEE. Trans. Ultrason., Ferroelect. Freq. Contr., vol. 50, pp. 1134-1146, Sep. 2003.

10. Q. Zhu and B. D. Steinberg, "Wavefront amplitude distribution in the female breast," J. Acoust. Soc. Am., vol. 96, pp. 1-9, Jul. 1994.

11. Y. Xie, B. Guo, L. Xu, J. Li, and P. Stoica, "Multi-static adaptive microwave imaging for early breast cancer detection," 39th ASILOMAR Conference on Signals, Systems and Computers, Pacific Grove, CA, October 2005.

12. M. Xu and L. V. Wang, "Time-domain reconstruction for thermoacoustic tomography in a spherical geometry," IEEE Trans. Med. Imag., vol. 21, pp. 814-822, Jul. 2002. 


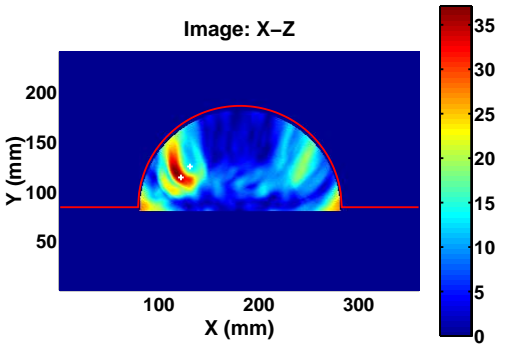

(a): DAS-PP

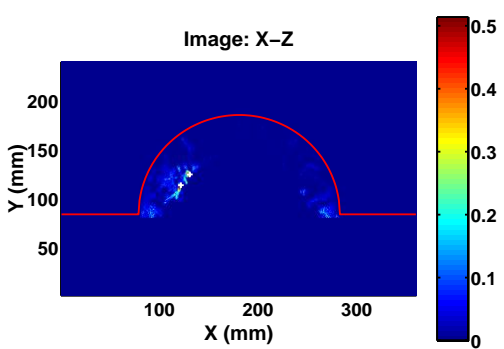

(d): ART-PP

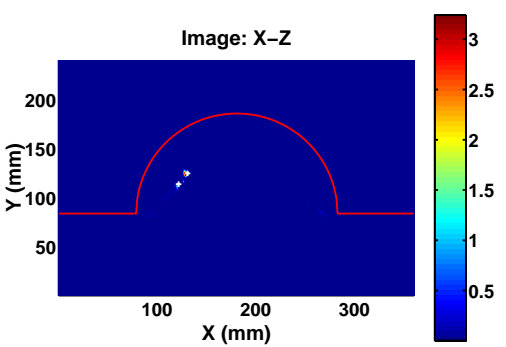

(b): RCB-E2 Image: $\mathbf{X - Z}$

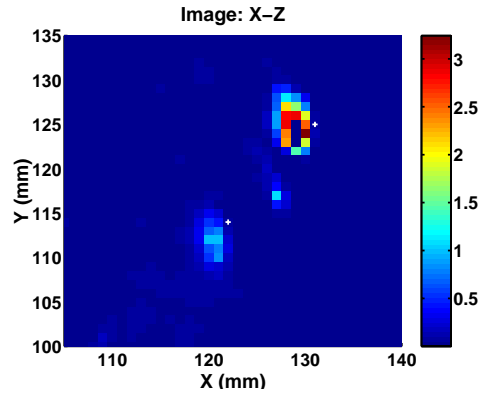

(e): Zoom in of (b)

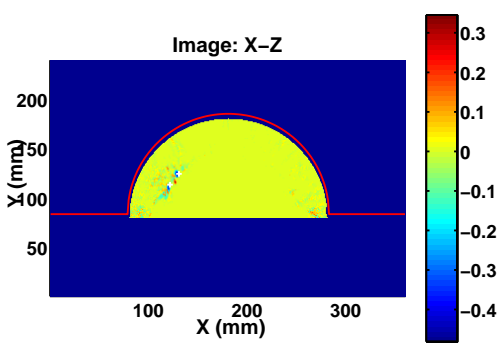

(c): ART-P

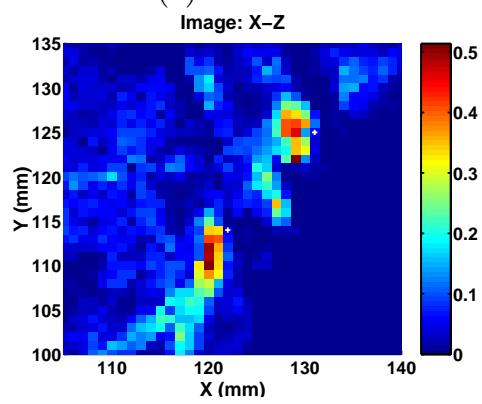

(f): Zoom in of (d)

Figure 2. Reconstructed images based on the 2-D simulated breast model, with two closely located (5mm spacing) small tumors (2 $\mathrm{mm}$ in diameter). The parameter used in RCB and ART is $\varepsilon=0.1 M$. The white dots in (e) and (f) correspond to the actual (center) location of the tumors. (a): DAS-PP; (b): RCB-E2; (c) ART-P; (d): ART-PP, (e): zoom in of RCB-E2; (f): zoom in of ART-PP.

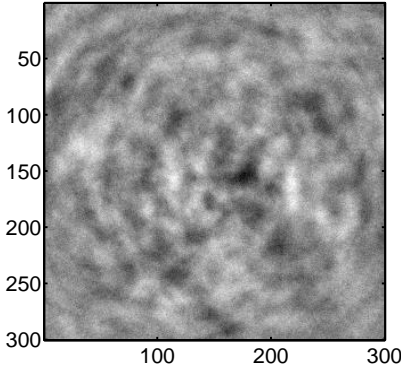

(a): DAS-C

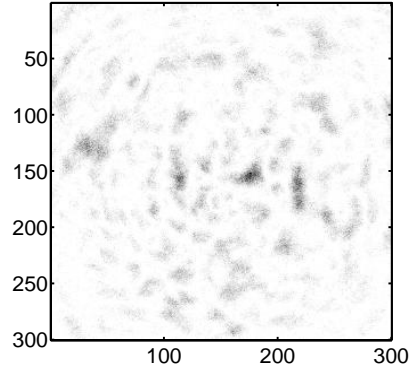

(b): DAS-E1 


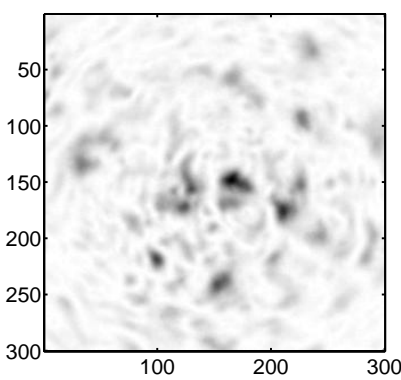

(c): DAS-E2

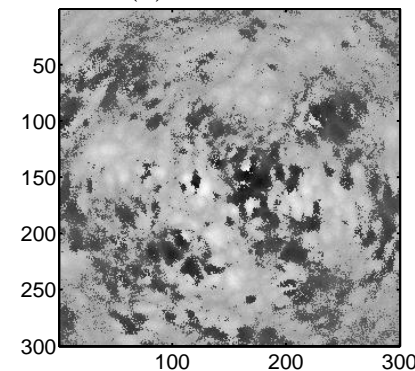

(e): DAS-P

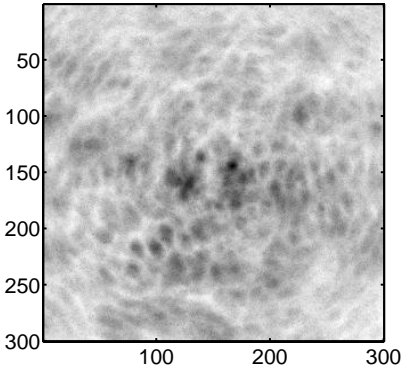

(g): DAS-PP

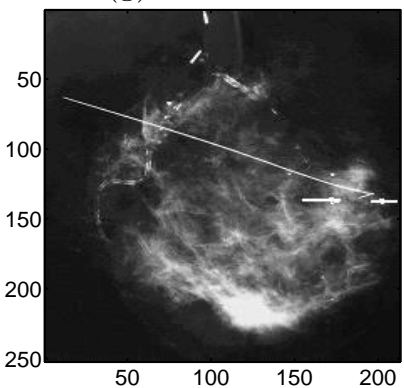

(i): X-ray image

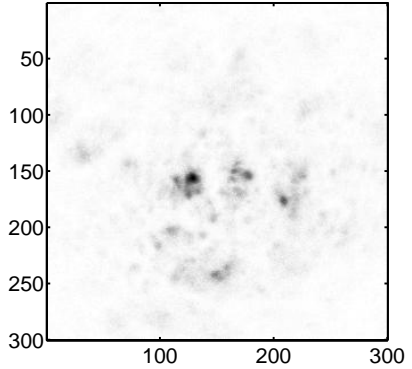

(d): RCB-E2

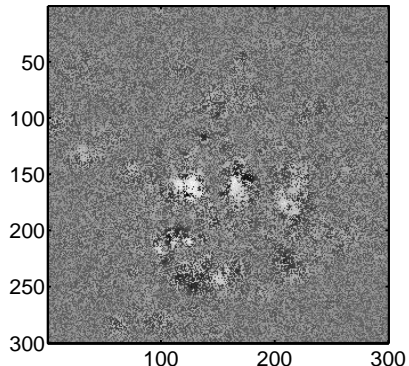

(f): ART-P

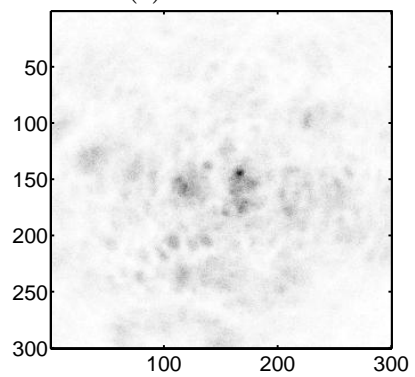

(h): ART-PP

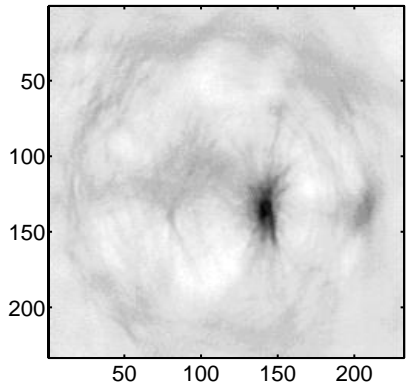

(j): Inverse Solution

Figure 3. Reconstructed images for breast specimen II. (a): DAS-C; (b): DAS-E1; (c) DAS-E2; (d): RCB-E2, with $\varepsilon=0.5 M ;(\mathrm{e}):$ DAS-P; (f): ART-P, with $\varepsilon=0.5 M ;(\mathrm{g}):$ DAS-PP; (h): ART-PP, with $\varepsilon=0.5 M ;(\mathrm{i}):$ X-ray image; (j): Inverse solution. 


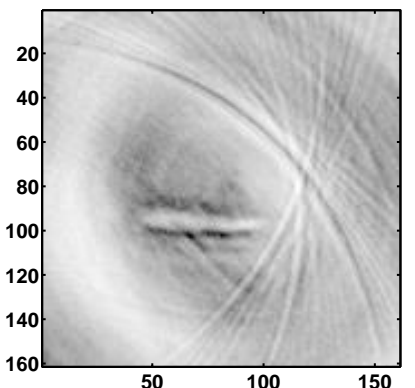

(a): DAS-C

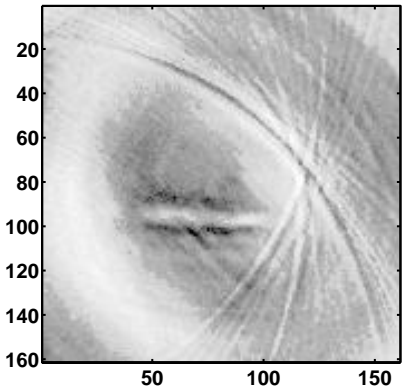

(c): DAS-P

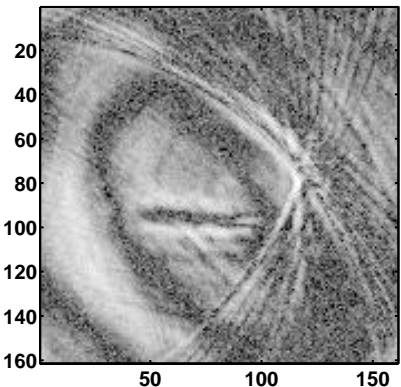

(e): DAS-PP

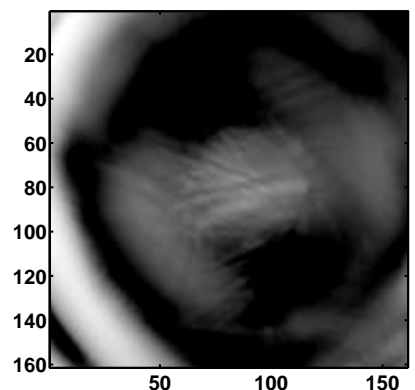

(b): RCB-E2

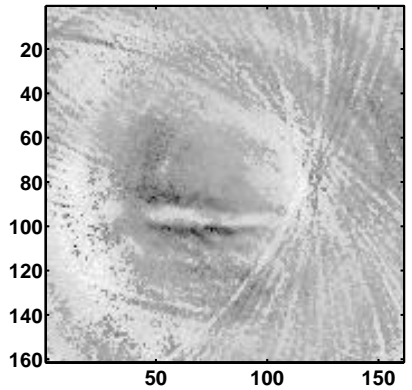

(d): ART-P

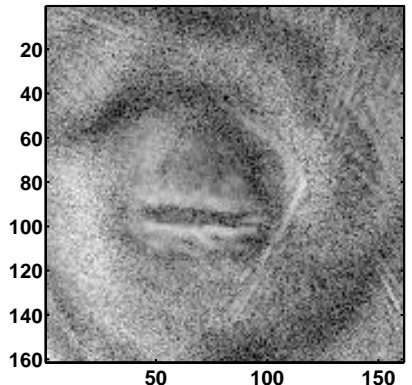

(f): ART-PP

Figure 4. Reconstructed images for blood object sample. The figures are normalized and displayed in logarithm scaled with dynamic range $15 \mathrm{~dB}$. (a): DAS-C; (b): RCB-E2; (c) DAS-P; (d): ART-P, with $\varepsilon=0.8 M$; (e): DAS-PP; (f): ART-PP, with $\varepsilon=0.8 M$. 